\title{
Constant-Stress Accelerated Degradation Life Test of an Organic Light-Emitting Diode Display under Violet Light
}

\author{
François-Xavier Fortier, Sylvain G. Cloutier \\ Department of Electrical Engineering, Ecole de Technologie Superieure, Montreal, Canada \\ Email: francois-xavier.fortier.1@ens.etsmtl.ca
}

Received 23 December 2015; accepted 3 February 2016; published 6 February 2016

Copyright (C) 2016 by authors and Scientific Research Publishing Inc.

This work is licensed under the Creative Commons Attribution International License (CC BY).

http://creativecommons.org/licenses/by/4.0/

(c) (i) Open Access

\begin{abstract}
The lifetime of commercial OLED display devices increases, so does the need for an accelerated lifetime testing method. The present work proposes a simple and accurate blackbox testing approach for commercial PMOLED display lifetime assessment using violet light-induced accelerated aging. Maximum likelihood estimations using lognormal distributions are performed based on datasets acquired from samples exposed to six different degrees of violet irradiance and accelerated life model is shown to accurately fit experimental data using an inverse power law. Based on these results, weighted average of the logarithmic standard deviation, the average life and median life can then be obtained for specific conditions of operation of the devices. As this method relies exclusively on violet light-induced degradation at room-temperature, this minimally-invasive testing procedure requires no significant modification to the display hardware architecture.
\end{abstract}

\section{Keywords}

PMOLED, Displays, Tests and Measurements, Lifetime, Light-Induced Aging, Accelerated Degradation Testing

\section{Introduction}

The organic light emitting diode (OLED) technology made reliable progress from its first viable prototypes in the late 1980's [1]. In recent years, a major issue has been to increase their lifetime. To do so, the main limitation factor remains as the blue light-emitting material, for which the half-life has been progressively increased from fewer than 800 hours to more than 20,000 hours in recent years [2] [3]. As the lifetime increases, it becomes essential for mass production to develop appropriate accelerated-aging testing methods to accurately pre- 
dict the displays time-of-life as fast as possible and at low costs. So far, most of the measurement techniques suggested involve direct current measurement across the junction [4]-[7]. Unfortunately, these methods require important hardware modifications and still require several hundreds of hours of testing. Recently, a new approach using ultra-violet (UV) light as a degradation agent was proposed as an efficient alternative mechanism for accelerated aging of OLEDs [8]. As such, this method requires no significant hardware modification prior to testing.

In this report, we use violet exposure from $405 \mathrm{~nm}$ LEDs as an accelerated-aging vector. While this method was previously used on home-made OLED devices, this report confirms that it can also be applied to fullypackaged commercial passive matrix OLED (PMOLED) displays. Indeed, we show that maximum likelihood estimation using lognormal statistics on data acquired from displays exposed to six (6) different violet irradiances can yield accurate accelerated-lifetime equations using a simple inverse power-law model. In the future, we believe this method can provide a simple, fast and low-cost accelerated-aging technique to yield accurate predictions for the weighted average of the logarithmic standard deviation, the average life and the median life for commercial OLED displays under specific operation conditions.

\section{Experimental Methods}

\subsection{The Commercial PMOLED Displays}

While UV-induced accelerated aging methods have been previously proposed [8], the main objective of this study is to demonstrate its potential to allow accurate lifetime prediction for commercial OLED displays. To do so, we used PMOLED displays (UG-9664HDDAG01) made by Univision Technology Inc. Shown in Figure 1, it has $96 \times 64$ RGB pixels and an active area of $20.14 \mathrm{~mm}$ by $13.42 \mathrm{~mm}$. It is mounted on a Waveshare Electronics PCB support to facilitate its integration and manipulation. Finally, the displays are powered and controlled using an Arduino Uno programmed with open-license libraries. The code was modified to generate a checkerboard pattern shown in Figure 1, as suggested by the ISO 92413052008 standardized testing method. As we will show later, this can allow simultaneous assessment of the influence of the violet light exposure on both the active and inactive regions of the displays. As OLED materials are fairly sensitive to light, the commercial PMOLED displays possess built-in UV filters shown in Figure 2(a). Using an Ocean Optics USB2000+ spectrometer connected to an integrating sphere, we measured the transmittance spectrum shown in Figure 2(b). The measured transmittance at $405 \mathrm{~nm}$ is $9.9 \%$.

\subsection{The Test Configuration}

As shown in Figure 3, high-power LEDs from LED Engin Inc. (LZ1-10UA00-00U8) having $1 \mathrm{~W}$ of radiant flux at $405 \mathrm{~nm}$ radiation are used to irradiate the displays. The irradiance of the $405 \mathrm{~nm}$ LEDs and the PMOLED are measured with a Thorlabs S120VC photodiode with its USB interface which has its own built-in calibration module. The PMOLED emission is measured at blue sub pixel peak value. The $405 \mathrm{~nm}$ excitation power can be

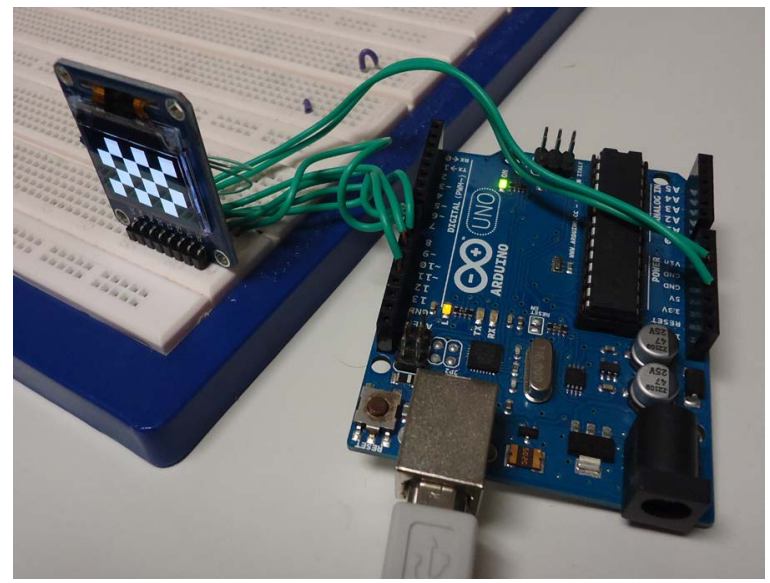

Figure 1. Commercial PMOLED displays made by Univision Technology Inc. with checkerboard pattern. 


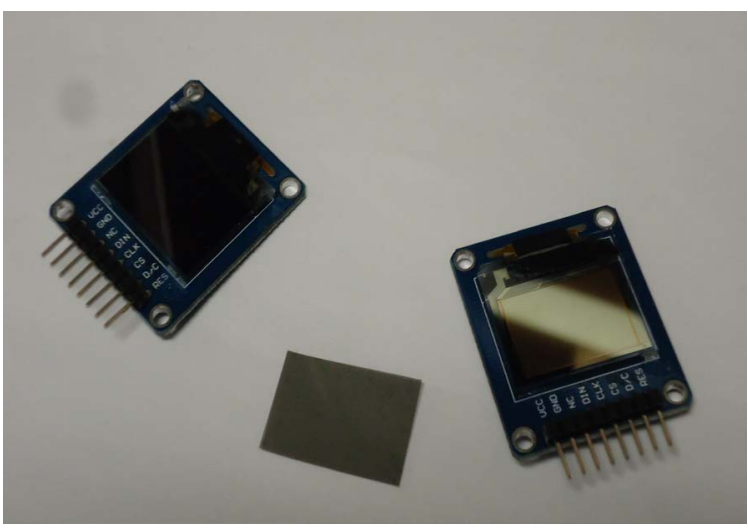

(a)

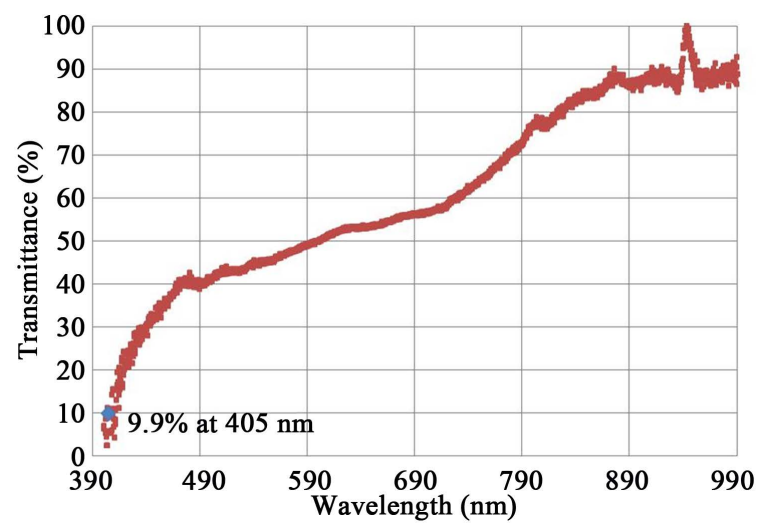

(b)

Figure 2. (a) PMOLED displays with and without the built-in UV filter; (b) Transmittance of the UV filter.

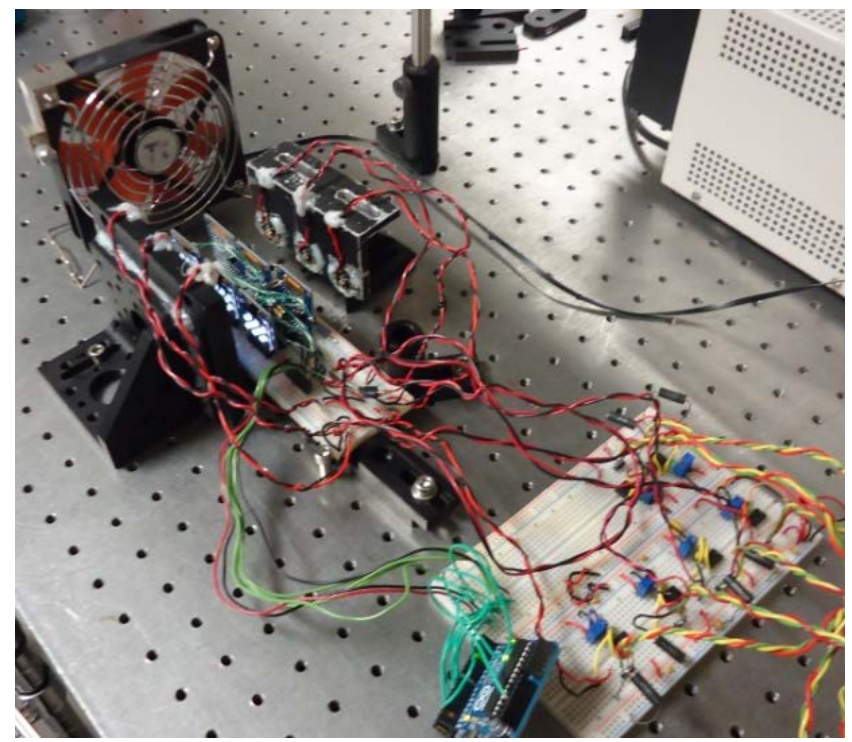

Figure 3. Violet light-induced accelerated-aging test configuration.

manually fine-tuned via an operational-amplifier with Darlington driver. As such, the $405 \mathrm{~nm}$ irradiance incident upon the display can be varied between 300 to $1100 \mathrm{~W} / \mathrm{m}^{2}$ to be consistent with the previously-proposed method [8].

An external fan is used to maintain the test bench at room-temperature to prevent the device from overheating and sustaining thermally-induced damage coupled with the violet light-induced aging. It also prevents the Darlington transistor from heating. Moreover, the whole setup was enclosed in a black box to avoid additional exposure to ambient lights.

During the accelerated aging, the emission from the display is measured every 24 hours following the test sequence previously proposed in [7]. The displays are considered to fail when they reach less than $50 \%$ of their initial power densities.

\subsection{The Time-Decay Model}

Féry et al. previously proposed a correlation between half-lifetime $\left(t_{0.5}\right)$ and the initial luminance $\left(L_{0}\right)$ [9]. This relation is given as

$$
t_{0.5}=\frac{C_{1}}{\left(L_{0}\right)^{n}}
$$


where $n$ and $C_{1}$ are characteristic parameters. Meanwhile, Seifert et al. also proposed a direct proportionality relationship between the initial luminance $\left(L_{0}\right)$ and the current density $(j)$ transforming this model into

$$
t_{0.5}=\frac{C_{2}}{(j)^{n}}
$$

where $n$ and $C_{2}$ are different characteristic parameters [8]. Finally, they propose to directly apply the same relationship between the current density across the device $(j)$ and the incident ultra-violet light power density $\left(I_{U V}\right)$ to yield

$$
t_{0.5}=\frac{C_{3}}{\left(I_{U V}\right)^{n}}
$$

where $n$ and $C_{3}$ are characteristic parameters [8].

\section{Results}

\section{The Commercial PMOLED Displays}

The data in Figure 4 shows the display power density decay as a function of time for different $405 \mathrm{~nm}$ irradiances. All data are normalized using the initial luminance $\left(L_{0}\right)$ at $t=0$.

In contrast, Figure 5 shows the half-lifetime $\left(t_{0.5}\right)$ extracted from the experimental data in Figure 4 as a function of the incident $405 \mathrm{~nm}$ irradiance. By fitting these results using Equation (3), we obtain the characteristic parameters $n=0.798$ and $C_{3}=19719$, which yields results similar to Seifert et al. [8].

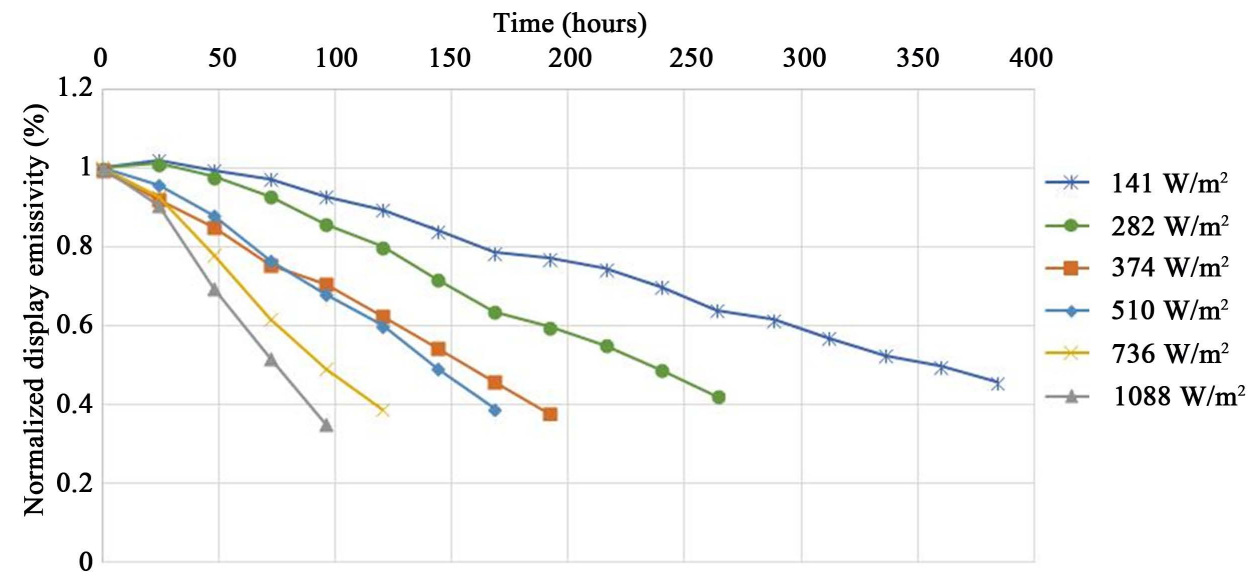

Figure 4. Degradation plot or the display power density normalized to the initial luminance $\left(L_{0}\right)$ as a function of the $405 \mathrm{~nm}$ exposure time for different irradiation in $\mathrm{W} / \mathrm{m}^{2}$.

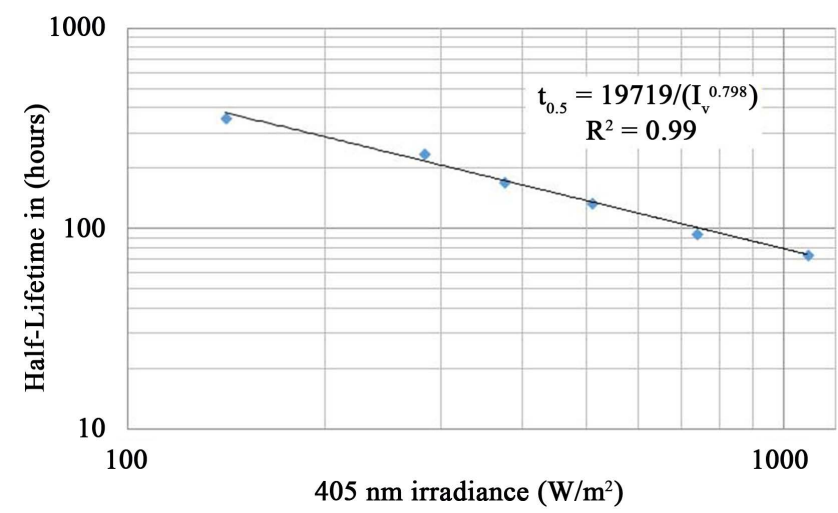

Figure 5. Half-lifetime $\left(t_{0.5}\right)$ as a function of the $405 \mathrm{~nm}$ exposure time for different irradiation power densities in $\mathrm{W} / \mathrm{m}^{2}$. 
While looking more closely at the origin of the display luminance decay, Figure 6(a) and Figure 6(b) show how the displays' RGB pixels are affected by the exposure to $405 \mathrm{~nm}$ light after reaching half-lifetime $\left(t_{0.5}\right)$. Meanwhile, Figure 6(c) shows the PMOLED displaying a white background after exposure to the $405 \mathrm{~nm}$ light. The exposure burnt the active checkerboard pattern. The whiter areas are those that were turned-off during the exposure test. This result clearly indicates that the $405 \mathrm{~nm}$ exposure affects significantly more the areas in the on-state during exposure.

\section{Analysis}

\subsection{Maximum Likelihood Estimation Using Lognormal Distributions}

According to Zhang et al., a WOLED satisfies a lognormal distribution and the maximum likelihood principles can be applied for calculation [5]. Here, we are assuming that a PMOLED would also have a similar ageing behavior. As such, the estimated logarithmic mean value $(\hat{\mu})$ and the estimated logarithmic standard deviation $(\hat{\sigma})$ can be expressed as

$$
\hat{\mu}=\frac{1}{n_{i}} \sum_{j=1}^{n_{i}} \ln \left(t_{j}\right)
$$

and

$$
\hat{\sigma}=\sqrt{\frac{1}{n_{i}-1} \sum_{j=1}^{n_{i}}\left(\ln \left(t_{j}\right)-\hat{\mu}\right)^{2}}
$$

where the $t_{j}$ are the failure times at stress levels $I_{V i}=(i=1,2,3, \cdots, 6)$ since we have 6 stress levels in this specific experiment. The three failure times at different stress levels $(j=1,2,3)$, are shown in Table 1 . The total number of samples at stress level $I_{V i}$ is $n_{i}$, and $n_{1,2,3, \cdots, 6}=3$.

Using Equations (4) and (5) with the information in Table 1, we can then calculate the estimated logarithmic mean value and the estimated logarithmic standard deviation extracted for each sample as shown in Table 2.

Meanwhile, the accelerated life equation would be an inverse power law

$$
\mu=\alpha+\beta \cdot \ln (I)
$$

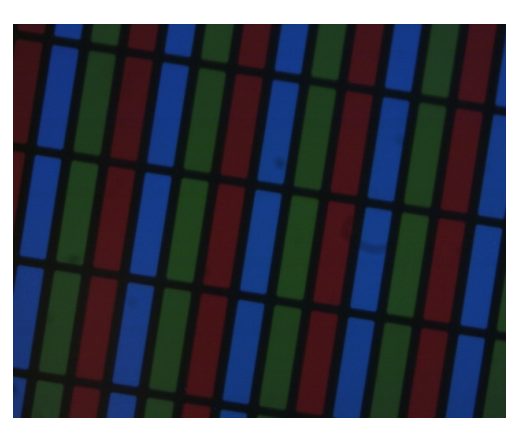

(a)

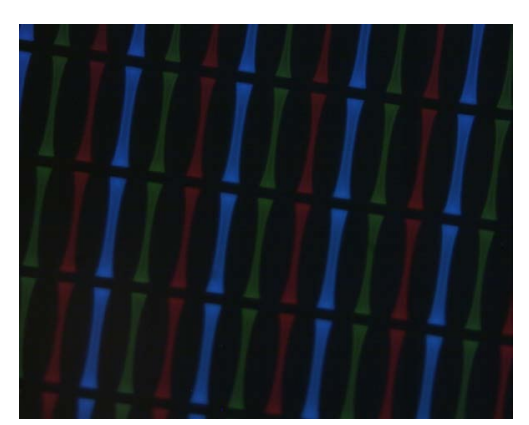

(b)

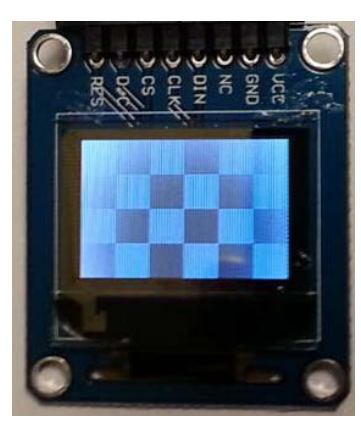

(c)

Figure 6. (a) RGB pixels before exposure; (b) RGB pixels affected by the exposure to $405 \mathrm{~nm}$ light after reaching half-lifetime $\left(\mathrm{t}_{0.5}\right)$; (c) PMOLED with a white background, showing the exposures burning effects.

Table 1. Failure times for each samples obtained through LSQ calculation of the raw data for each display.

\begin{tabular}{ccccccc}
\hline \multirow{2}{*}{$\begin{array}{c}\text { Failure times } \\
\text { (hours) }\end{array}$} & $I_{V 1}=141.08$ & $I_{V 2}=282.16$ & $I_{V 3}=374.03$ & $I_{V 4}=510.04$ & $I_{V 5}=736.73$ & $I_{V 6}=1088.09$ \\
\cline { 2 - 7 } & 377.29 & 243.71 & 167.56 & 136.80 & 94.09 & 76.06 \\
$t_{1}$ & 366.51 & 246.04 & 156.07 & 137.92 & 96.64 & 76.09 \\
$t_{2}$ & 336.59 & 230.00 & 194.29 & 135.11 & 94.78 & 72.79 \\
\hline
\end{tabular}


where $\alpha$ and $\beta$ are the acceleration parameters and $I$ is the stress intensity [5]. Here, the intensity of the $405 \mathrm{~nm}$ light $I_{V}=I$. This model is perfectly consistent with the experimental data shown in Figure 7, from which we can extract the acceleration parameters $\alpha=9.8944$ and $\beta=-0.7997$.

\subsection{Average Lifetime Estimation}

As such, the weighted average of the logarithmic standard deviation, the average life and median life can be given as

$$
\hat{\sigma}_{0}=\frac{\sum_{i=1}^{6} n_{i} \hat{\sigma}_{i}}{\sum_{i=1}^{6} n_{i}}
$$

and

$$
\bar{\mu}=\exp \left(\mu_{0}+\frac{1}{2} \hat{\sigma}_{0}^{2}\right)
$$

and

$$
t_{0.5}=\exp \left(\mu_{0}\right)
$$

where $\hat{\sigma}_{0}$ is the weighted average of $\hat{\sigma}$ values in Table 2, which gives the estimated standard deviation to be $\hat{\sigma}=0.043$. To solve Equation (8) and Equation (9), the logarithmic mean value $\mu_{0}$ is approximated by $\mu$ as given by Equation (6).

Obviously, the real device lifetime statistics will depend heavily on the environment in which the devices are used. As such, accurate predictions require an appropriate value for the irradiance $I$ depending on the conditions in which the displays will be used. As an example, let us assume a display device that would be used in an environment subjected to direct sunlight exposure. There, $I$ at $405 \mathrm{~nm}$ would be equal to $0.8785 \mathrm{~W} / \mathrm{m}^{2} / \mathrm{nm}$ at direct

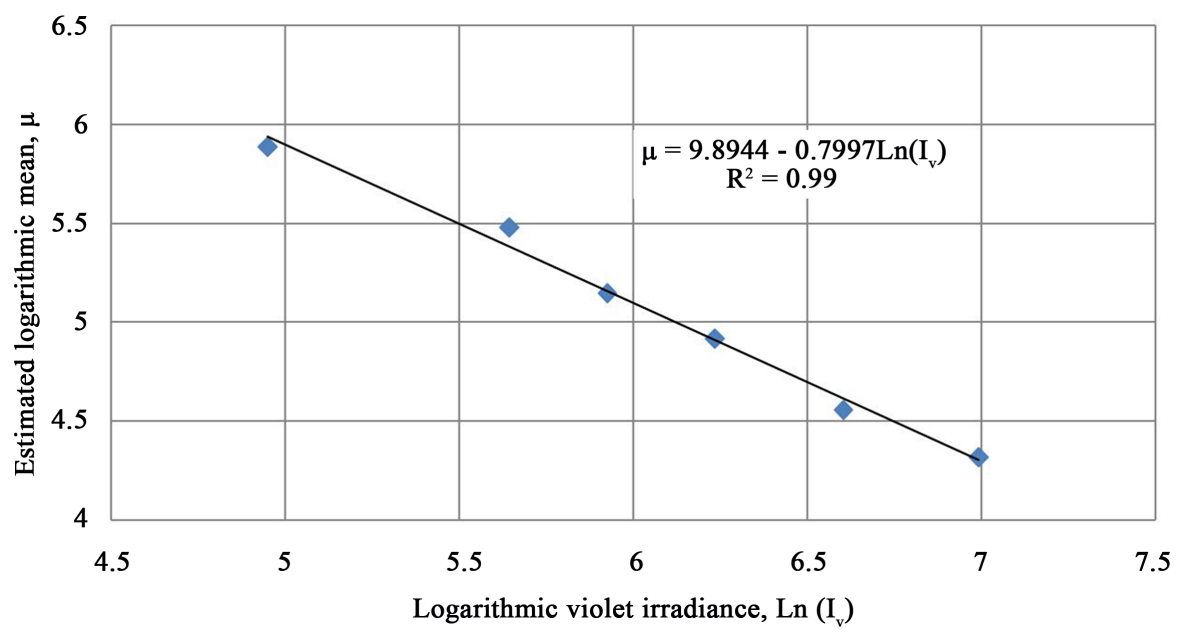

Figure 7. Parametric lognormal representation and fit using an inverse power law. Using a fit, we can get the $\alpha$ and $\beta$ for Equation (6).

Table 2. Estimated logarithmic mean values and estimated logarithmic standard deviation for each samples obtained through LSQ calculation of the raw data for each display.

\begin{tabular}{ccccccc}
\hline & \multicolumn{5}{c}{ Violet light irradiance $\left(I_{V}\right)$} \\
\cline { 2 - 7 } & $I_{V 1}=141.08$ & $I_{V 2}=282.16$ & $I_{V 3}=374.03$ & $I_{V 4}=510.04$ & $I_{V 5}=736.73$ & $I_{V 6}=1088.09$ \\
$\hat{\mu}$ & 5.885 & 5.48 & 5.147 & 4.917 & 4.569 & 4.317 \\
$\hat{\sigma}$ & 0.059 & 0.036 & 0.112 & 0.010 & 0.024 & 0.025 \\
\hline
\end{tabular}


normal circumsolar irradiance (according to standard ASTM G173-03) into Equation (6) and thus obtain $\mu_{0} \cong \mu=9.998$. Submitting the results of Equation (6) and Equation (7) into Equation (8), we found the average life $\bar{\mu}=22003$ hours. Since $\mu_{0} \cong \mu=9.998$, the median life from Equation (9) is $t_{0.5}=21982.5$ hours. Naturally, the conditions given in standard ASTM G173-03 can't be applied to every situation where the PMOLED or OLED device will be used and are used here only as an example.

\section{Conclusion}

Maximum likelihood estimations using lognormal distributions are performed based on datasets acquired from samples exposed to six different degrees of violet irradiance and accelerated life model is shown to accurately fit experimental data using an inverse power law. Based on these results, weighted average of the logarithmic standard deviation, the average life and median life can then be obtained for specific conditions of operation of the devices. Given a certain light irradiance as reference for a representative lifetime of the OLED device, the lifetime statistics for an OLED device can be predicted with the method shown in this study. Most importantly, no alteration to the device is needed to perform this accelerated degradation test.

\section{Acknowledgements}

S.G.C. is most thankful for the financial support from the Canada Research Chairs and the NSERC Discovery programs.

\section{References}

[1] Tang, C.W. and Van Slyke, S.A. (1987) Organic Electroluminescent Diodes. Applied Physics Letters, 51, 913-915. http://dx.doi.org/10.1063/1.98799

[2] Laaperi, A. (2008) OLED Lifetime Issues from a Mobile-Phone-Industry Point of View. Journal of the Society for Information Display, 16, 1125-1130. http://dx.doi.org/10.1889/JSID16.11.1125

[3] Mertens, R. (2014) The OLED Handbook: A Guide to OLED Technology, Industry \& Market. OLED-Info.

[4] Park, J.I. and Bae, S.J. (2010) Direct Prediction Methods on Lifetime Distribution of Organic Light-Emitting Diodes from Accelerated Degradation Tests. IEEE Transactions on Reliability, 59, 74-90. http://dx.doi.org/10.1109/TR.2010.2040761

[5] Zhang, J., Liu, F., Liu, Y., Wu, H., Wu, W. and Zhou, A. (2012) A Study of Accelerated Life Test of White OLED Based on Maximum Likelihood Estimation Using Lognormal Distribution. IEEE Transactions on Electron Devices, 59, 3401-3404. http://dx.doi.org/10.1109/TED.2012.2215864

[6] Zhang, J., Liu, C., Cheng, G., Chen, X., Wu, J., Zhu, Q. and Zhang, L. (2014) Constant-Stress Accelerated Life Test of White Organic Light-Emitting Diode Based on Least Square Method Under Weibull Distribution. Journal of Information Display, 15, 71-75. http://dx.doi.org/10.1080/15980316.2014.889613

[7] Zhang, J., Li, W., Cheng, G., Chen, X., Wu, H. and Herman Shen, M.-H. (2014) Life Prediction of OLED for ConstantStress Accelerated Degradation Tests Using Luminance Decaying Model. Journal of Luminescence, 154, 491-495. http://dx.doi.org/10.1016/j.jlumin.2014.05.024

[8] Seifert, R., Scholz, S., Lüssem, B. and Leo, K. (2010) Comparison of Ultraviolet- and Charge-Induced Degradation Phenomena in Blue Fluorescent Organic Light Emitting Diodes. Applied Physics Letters, 97, Article ID: 013308. http://dx.doi.org/10.1063/1.3460285

[9] Féry, C., Racine, B., Vaufrey, D., Doyeux, H. and Cinà, S. (2005) Physical Mechanism Responsible for the Stretched Exponential Decay Behavior of Aging Organic Light-Emitting Diodes. Applied Physics Letters, 87, Article ID: 213502. http://dx.doi.org/10.1063/1.2133922 